\title{
Head and neck injury potential during water sports falls: examining the effects of helmets
}

\author{
Irving S. Scher ${ }^{1}\left[\right.$ [ $\cdot$ Lenka L. Stepan ${ }^{1} \cdot$ Ryan W. Hoover ${ }^{2}$
}

Published online: 16 March 2020

(c) The Author(s) 2020

\begin{abstract}
Head and neck injuries sustained during water skiing and wakeboarding occur as a result of falls in water and collisions with obstacles, equipment, or people. Though water sports helmets are designed to reduce injury likelihood from head impacts with hard objects, some believe that helmets increase head and neck injury rates for falls into water (with no impact to a solid object). The effect of water sports helmets on head kinematics and neck loads during simulated falls into water was evaluated using a custom-made pendulum system with a Hybrid-III anthropometric testing device. Two water entry configurations were evaluated: head-first and pelvis-first water impacts with a water entry speed of $8.8 \pm 0.1 \mathrm{~m} / \mathrm{s}$. Head and neck injury metrics were compared to injury assessment reference values and the likelihoods of brain injury were determined from head kinematics. Water sport helmets did not increase the likelihood of mild traumatic brain injury compared to a non-helmeted condition for both water entry configurations. Though helmets did increase injury metrics (such as head acceleration, HIC, and cervical spine compression) in some test configurations, the metrics remained below injury assessment reference values and the likelihoods of injury remained below $1 \%$. Using the effective drag coefficients, the lowest water impact speed needed to produce cervical spine injury was estimated to be $15 \mathrm{~m} / \mathrm{s}$. The testing does not support the supposition that water sports helmets increase the likelihood of head or neck injury in a typical fall into water during water sports.
\end{abstract}

Keywords Wakeboarding $\cdot$ Water skiing $\cdot$ Head injury $\cdot$ Cervical spine injury $\cdot$ Head protection $\cdot$ Bucketing; Venting

\section{Introduction}

During water skiing and wakeboarding, a participant rides on top of the water while being towed behind a boat or by ropes attached to a rotating cableway located well above the water surface in a water sports park (called "cable wakeboarding"). The Sports and Fitness Industries Association estimated that in 2016, there were 3.7 million. participants for water skiing and 2.9 million. participants for wakeboarding in the United States [1]. As with many popular

Irving S. Scher

scher@guidanceengineering.com

Lenka L. Stepan

stepan@guidanceengineering.com

Ryan W. Hoover

rhoover@imminet.com

1 Guidance Engineering and Applied Research, 205 NE Northlake Way, Suite 100, Seattle, WA 98105, USA

2 CAPE, 18881 IMMI Way, Westfield, IN 46074, USA recreational activities, water skiers and waterboarders are at risk of sustaining serious injuries.

Epidemiology articles have examined the prevalence of head and neck injuries from water skiing and wakeboarding. Carson [2] surveyed 156 orthopedic surgeons and 86 wakeboarders to obtain wakeboarding injury data. A total of 82 injuries were reported by 66 wakeboarders; $26 \%$ of the injuries involved the head, back, or ribs. Loughlin [3] reported that $7.5 \%$ of the injuries sustained in competition water skiing were to the head and neck. Hostetler et al. [4] analyzed data from the National Electronic Injury Surveillance System (NEISS) for water skiing and wakeboardingrelated injuries treated in United States emergency departments from 2001 to 2003. Head injuries and traumatic brain injuries represented $4.3 \%$ and $2.4 \%$ of all injuries to water skiers and $28 \%$ and $12.5 \%$ of all injuries to wakeboarders. Baker et al. [5] used data from the NEISS database collected between 2000 and 2007 to compare injuries sustained during wakeboarding, water skiing, and water tubing in the United States. Of 1761 individual emergency room visits, the head and neck were the most commonly injured body region 
during wakeboarding and tubing and the second most commonly injured body region during water skiing. The second most common diagnosis for the head and neck region was a concussion. Schofer et al. [6] investigated injury rates for cable wakeboarding during a 6-month season in Germany. They found that $77.6 \%$ of injuries occurred during jumps on kickers, ramps, and fun boxes and observed the rate of injury to increase with ability level. They reported ten concussions resulting from water impacts for individuals who were not wearing helmets. The prevalence (percent of all injuries) for head and neck injuries is high in wakeboarding and waterskiing $[5,6]$.

Case studies have also documented head and neck injuries from water sports. Carson [2] indicated that the majority of wakeboarding injuries occurred during falls while attempting a maneuver and reported a case study in which an unhelmeted wakeboarder sustained a subdural hematoma while attempting a flip. Hummel and Gainor [7] presented cases of facial and scalp lacerations from falls and a fractured skull from a towrope-related accident. Chia et al. [8] reported a case study in which a wakeboarder experienced a highspeed, face-first fall onto the water, producing an intracranial hemorrhage. Kluger and Jegou [9] reported a case study on a neck laceration sustained during cable-tow skiing. Though head and neck injuries during water skiing and wakeboarding have been reported from falls into water (without solid object contact), collisions with solid objects (such as buoys, ramps, docks, skis, or wakeboards), towrope entanglements, and contact with boat propellers [2-9], there are no data categorizing the prevalence of these injury scenarios.

Based on the epidemiologic data, head and neck injuries represent a major portion of the severe injuries sustained by water sports participants. Because some members of the water sports community have suggested water sports helmet use as a strategy to prevent head injuries, many cabletow water sports parks in the United States now require that their customers wear helmets. While many researchers agree that helmets would be effective at mitigating the injury potential during a head impact with a fixed object or water sports equipment, critics argue that helmets increase the likelihood of severe brain and cervical spine injuries during a fall into water; there are no epidemiologic data that classify injuries as either solid object or water only contact. Chia et al. [8] suggested that the increased cross-sectional area of a helmeted head would increase the resistance in the water, resulting in larger deceleration forces on the head and neck. In the same vein, water entering the helmet cavity during a backward fall could allow the helmet to act as a drogue or parachute anchor (termed "bucketing"). In this scenario, when the back edge of the helmet contacts water, the water enters the space between the head and the helmet and creates an imbalance between inflow momentum and outflow momentum. Two types of severe injuries from bucketing could occur by the helmet slowing more rapidly than the head and applying a superiorly directed force to the head through the chinstrap: (1) brain injuries from increased head acceleration and (2) cervical spine injuries from increased force and moment. While this phenomenon can cause severe cervical spine injuries during high-speed military aircraft ejections (that occur in air, not water) [10], the authors know of no peer-reviewed, published study that has determined the magnitude of this effect in water for water sports helmets at speeds typical of water skiers and wakeboarders.

Two previous studies examined the effects of helmets during water entry and deceleration. Taylor et al. [11] examined the effects of helmets during water entry by conducting drop tests into water using a Hybrid-III 50th percentile male anthropomorphic test device (ATD) fitted with helmets (open-face and full-face bicycle) and without helmets. The data from these tests were used to estimate the effects at higher speed using a hydrodynamic model. Using this model, Taylor et al. predicted that cervical spine injuries would occur at lower water entry speeds when a person was wearing a helmet. Unfortunately, the tests and hydrodynamic modeling did not use water sports helmets and there are limited data provided to reproduce this study. Sheer et al. [12] conducted a laboratory study to examine the effect of helmet surface geometry on the linear acceleration of the head during water impacts. In their testing, Sheer et al. dropped a Hybrid-III ATD head wearing an American football helmet (with fins and dimples added) into a small tank of water from $1.85 \mathrm{~m}$. The water impact speed in this study was approximately $6 \mathrm{~m} / \mathrm{s}$, below the speeds of water skiers towed behind watercraft [13] and wakeboarders at cable-tow parks $(9 \mathrm{~m} / \mathrm{s})$. It is unclear how either of these studies translate to helmets used in water sports and actual fall configurations of water sports participants because they used helmets designed for different sports, the methods may have edge effects (due to a small tank size), and many of the tests were at speeds below those of typical water skiers and wakeboarders.

To determine whether water sports helmets increase the likelihood of head and cervical spine injuries, further epidemiologic studies are necessary. This would require more data for injured and non-injured water skiers and wakeboarders than are collected currently, as well as exposure data. Because these data are not available, the research presented in this article attempts to shed light on the influence of a water sports helmet on the likelihood of brain and cervical spine injuries during water impacts similar to those seen in forward and backward falls during wakeboarding and water skiing. To test the effect of water sport helmets in these fall scenarios, a laboratory model (instead of instrumenting water sports participants) was used because of safety concerns with repeated head impacts and the challenges in producing comparable falls with and without helmets. 


\section{Materials and methods}

A custom-built pendulum was used to accelerate the head, neck, torso, and pedestrian pelvis of a Hybrid-III 50thpercentile male anthropomorphic test device (ATD) into a pool of water; see Fig. 1. The impact orientation (the angle of water entry and direction) was adjustable by modifying the position and length of steel cables that ran from the pendulum fulcrum to the ATD. The impacts were designed to replicate a backward fall while facing toward or away from the main direction of motion (that is, a fall while facing forward or backward). Tests were run with and without helmets, as detailed below.

\subsection{Testing equipment}

The ATD had a seated height of $88 \mathrm{~cm}$, was ballasted to weigh $60 \mathrm{~kg}$ (with a $4.5 \mathrm{~kg}$ head) without clothing, and wore a 2-mm spring wetsuit for all tests. The ATD was instrumented to quantify head accelerations and cervical spine loads during water entry. Linear, triaxial accelerometers (Endevco, Model No. 7264B-2000, San Juan Capistrano, California, USA) were mounted at the center of mass of the ATD head; the range of the accelerometers was $\pm 500 \mathrm{Gs}$ (accuracy: 0.25 Gs) along each axis. Angular velocity sensors (DTS, Model ARS, Seal Beach, California, USA) measured the rotation rate of the head; the range was $\pm 26.2 \mathrm{rad} / \mathrm{s}$ (accuracy: $0.17 \mathrm{rad} / \mathrm{s}$ ) along each axis. A six-axis, upper neck load cell (Denton Model No. 1716, Plymouth,

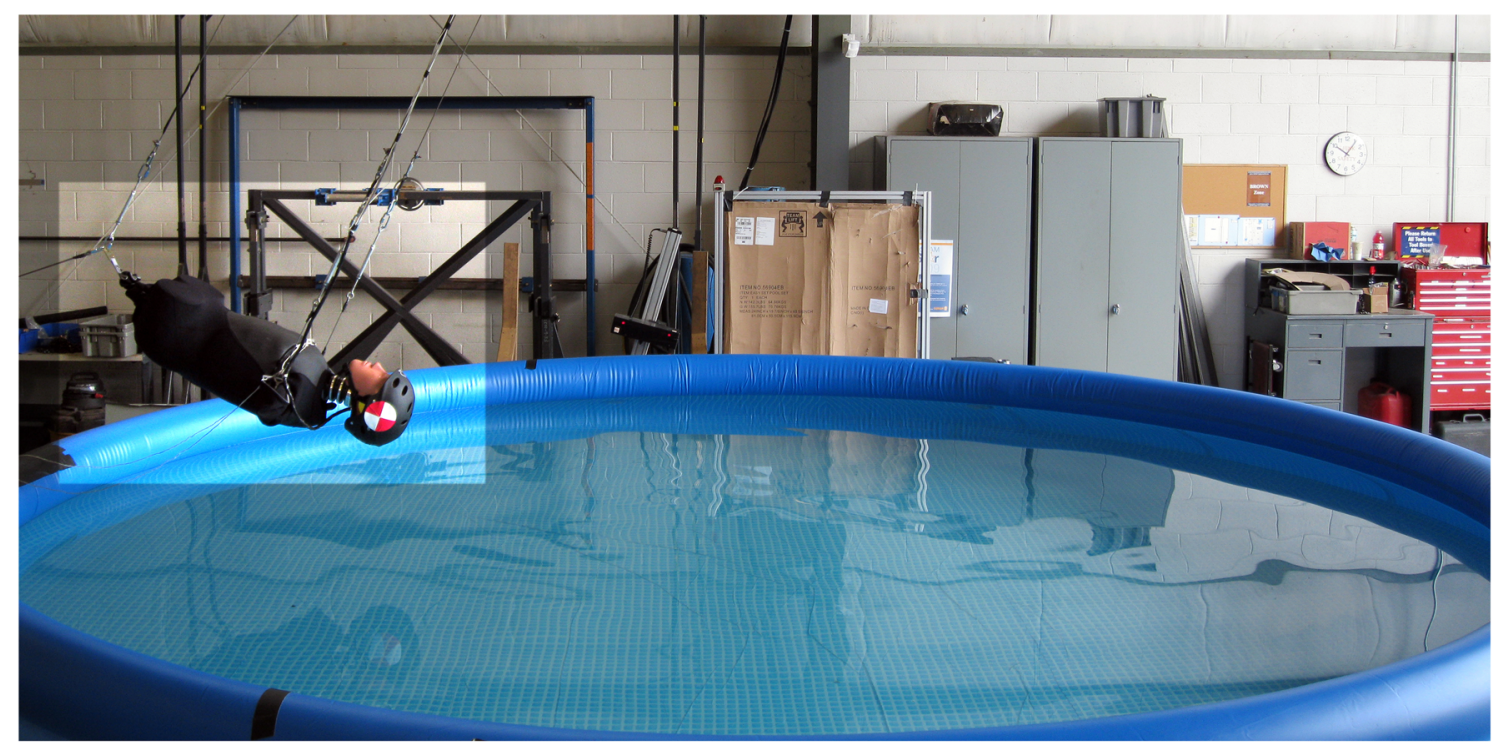

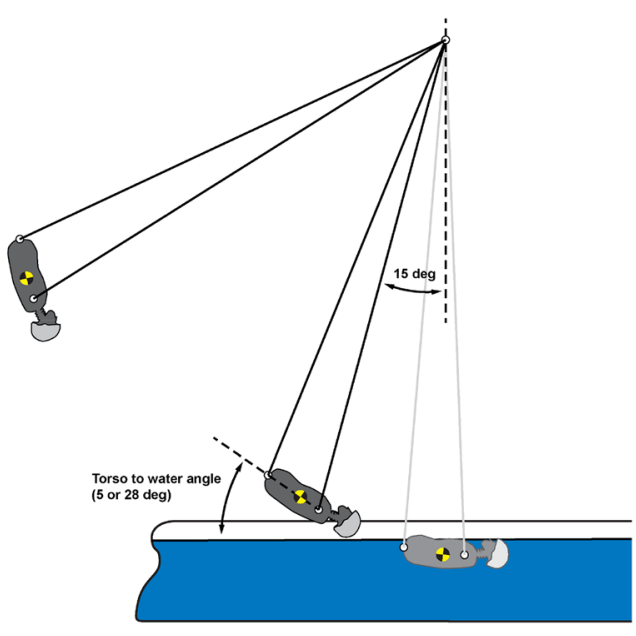

Fig. 1 (Top) Head-first water impact into the 5.5-m-diameter pool. (Bottom) Schematic of the testing setup used for the water impact tests: (Bottom-Left) Head-First impact configuration; in this configuration, two torso-to-water angles were tested, $5^{\circ}$ and $28^{\circ}$. (Bottom-

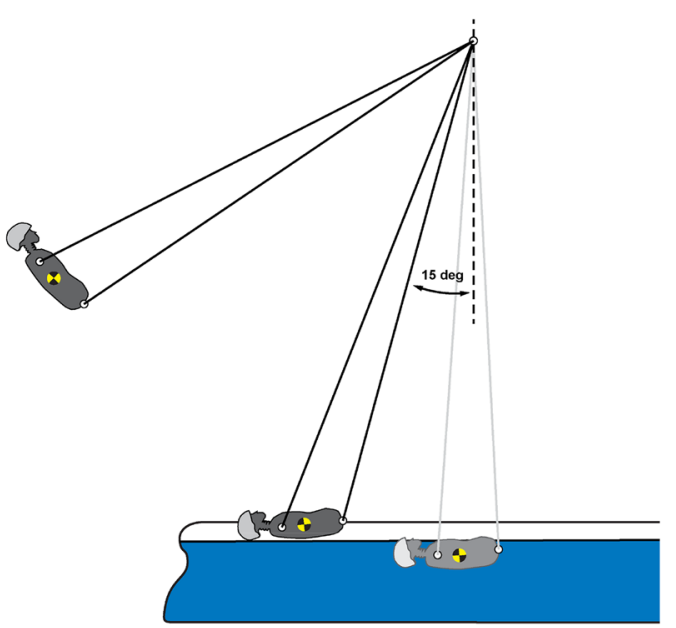

Right) Pelvis-First impact configuration with a torso-to-water angle of $11^{\circ}$. In both configurations, the ATD was raised upward along the pendulum arc and released, allowing it to swing freely and contact the water 
Michigan, USA) measured the forces and torques on the neck. The upper neck load cell had a range of $\pm 8896 \mathrm{~N}$ of force (accuracy: $4.5 \mathrm{~N}$ ) along the anterior-posterior and left-right axes, $\pm 13,345 \mathrm{~N}$ of force (accuracy: $6.7 \mathrm{~N}$ ) along the superior-inferior axis, and $\pm 282.5 \mathrm{Nm}$ of torque (accuracy: $1.4 \mathrm{Nm}$ ) along each axis. All instrumentation was sealed to prevent water intrusion. Each channel was lowpass filtered using a $2500-\mathrm{Hz}$ anti-aliasing filter and digitally sampled at 10,000 Hz.

The ATD speed just prior to water impact was measured using a laser speed trap (Messring, HB12-11-00, Germany); the accuracy was $0.01 \mathrm{~m} / \mathrm{s}$. Each test trial was recorded using real-time video and high-speed video (1000 frames per second; $1280 \times 800$ pixels; Vision Research, Phantom V210, New Jersey) synchronized using a direct cable connection with the ATD data acquisition system.

Two water sport helmet models representative of water sports helmets sold in the United States and one bicycle helmet (labeled A through C) were used in the testing; see Fig. 2; the details for each helmet are listed in Table 1. The two water sports helmets spanned the range of vents available, with Helmet A having the most venting (by area and number) and Helmet B having no vents. Each helmet was sized appropriately for the ATD head and was adjusted to fit the headform according to the manufacturers' instructions. Fit was verified by ensuring that there was little relative motion between the helmet and headform during manual manipulation of the helmet; each helmet could move upward $2 \mathrm{~cm}$ or less during manual manipulation.

\subsection{Protocol and impact configurations}

The procedure for creating water entry was the same for each test trial. The helmeted (or non-helmeted) ATD was raised upward along the pendulum arc and released, allowing it to swing freely and contact the water at $8.8 \mathrm{~m} / \mathrm{s}$; our impact speed was limited by the height of the test facility but was similar to wakeboarder speeds in cable-tow parks. After the ATD contacted the water, the pendulum cables went slack (no tension) and the ATD moved through the water unconstrained. This allowed the ATD to be submerged during the deceleration phase. To limit pool edge and depth effects, the ATD's initial water entry point was at least $1.75 \mathrm{~m}$ from the edge of the 5.5-m-diameter pool and the water depth was maintained to be at least $1 \mathrm{~m}$; the contact point was checked for each test using the high-speed video.

Test trials were conducted without a helmet and with the three helmet models. Based on the injury mechanisms described in the previous papers [2,8], a review of injury data from the United States Consumer Products Safety Commission (used in $[4,5]$ ), and the kinematics of edge catches from similar activities (snowboarding in [14]), a Head-First impact scenario was tested for each helmet condition. The Head-First impact configuration simulated a scenario in which a water skier or wakeboarder gets turned backward relative to the direction of travel and falls backward into the water with the superior, occipital region of the head leading; see Fig. 1-Bottom-Left. For this Head-First impact configuration, the ATD torso angle relative to the water surface


Fig. 2 (Left-to-Right) Helmet models A-C used for testing as worn by the ATD. Fit was verified by ensuring that there was little relative motion between the helmet and headform during manual manipulation of the helmet

Table 1 Helmet characteristics

\begin{tabular}{lllllr}
\hline Helmet & Helmet type & Liner materials & Standards met or exceeded $^{\mathrm{a}}$ & Weight $(\mathrm{kg})$ & Vents \\
\hline A & Water sport & Ethylene vinyl acetate & CE EN1385 [26] & 0.33 & 15 \\
B & Water sport & Brock foam & CE EN1385 [26] & 0.54 & 0 \\
C & Bicycle & Expanded polystyrene & CE EN1078 [32] & 0.34 & 21 \\
\hline
\end{tabular}

${ }^{a}$ According to the label inside the helmet

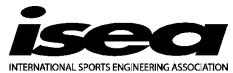


at impact was either $5^{\circ}$ or $28^{\circ}$, providing two testing conditions: (1) Head-First, Shallow and (2) Head-First, Inclined.

Based on the observations of water sports falls, a third ATD water impact configuration (Pelvis-First) was tested for each helmet condition that provided an opportunity for the helmet to act as a drogue (or parachute anchor). The PelvisFirst impact configuration simulated a scenario in which the water skier or wakeboarder is facing the direction of travel and falls backward, contacting the water surface with the occipital region of the head; see Fig. 1-Bottom-Right. Based on pilot testing, the torso angle at water entry was kept at $11^{\circ}$ (that is, with the pelvis above the water surface when the head contacted the water surface) to maximize the potential for water to flow into the helmet and act as a drogue. At least three tests were conducted for each combination of helmet and impact configuration.

\subsection{Data processing}

For each test trial, the data from the accelerometers and rate gyroscopes were filtered digitally using $1650-\mathrm{Hz}$ and 300-Hz low-pass filters (in accordance with SAE standard J211-1, Rev. March 2014 [15] and Bussone et al. [16] and confirmed by a residual analysis). Angular acceleration data were calculated by differentiating the angular rate data. For each trial, the Head Injury Criterion (HIC) [17] value that correlates with the likelihood of skull fracture and severe brain injury was calculated using:

$\mathrm{HIC}=\left[\frac{1}{t_{1}-t_{2}} \int_{t_{1}}^{t_{2}} a \mathrm{~d} t\right]^{2.5}\left(t_{2}-t_{1}\right)$,

where $a$ is the resultant head acceleration, and $t_{1}$ and $t_{2}$ were chosen over a $15-\mathrm{ms}$ interval such that the equation was maximized. In addition, the linear acceleration and angular rate data were integrated to determine the linear velocity and neck flexion angle for the first $350 \mathrm{~ms}$ after water impact; this time interval captured the peak loads and kinematics for all tests.

For each trial, the maximum resultant linear and angular head accelerations were ascertained; these metrics were compared to the Injury Assessment Reference Value (IARV) of $180 \mathrm{Gs}$ [18] and the level typically associated with rotational brain injury of $10,000 \mathrm{rad} / \mathrm{s}^{2}[19,20]$. The likelihoods of mild traumatic brain injury (mTBI) were determined using the risk curves based on the combination of peak resultant linear and angular accelerations [21]. For each trial, the maximum cervical spine compression, tension, flexion moment, and extension moment were ascertained; these metrics were compared to the IARVs that correspond to a $3 \%$ risk of serious injury [18].
Likelihoods of head and neck injury were determined using the risk curves from Mertz et al. [18] and Rowson and Duma [21].

To compare the effects of the helmet conditions, the drag force parallel to the direction of motion was calculated for each time step using:

$F_{D}=\left(m_{h} \cdot a_{x}-F_{x}\right) \sin \left(\theta_{Y}\right)-\left(m_{h} \cdot a_{z}-F_{z}\right) \cos \left(\theta_{Y}\right)$,

where $F_{D}$ is the drag force; $m_{h}$ is the mass of the ATD headform, $a_{X}$ and $F_{X}$ are the anterior-posterior head acceleration and neck load components; $a_{Z}$ and $F_{Z}$ are the superior-inferior head acceleration and neck load components; and, $\theta_{Y}$ is the flexion-extension angle of rotation. The drag force was used to determine the effective (total) drag coefficient, $C_{T}$, for each helmet using:

$F_{D}=\frac{C_{D} A \rho v^{2}}{2}=C_{T} v^{2}$,

where $C_{D}$ is the geometric drag coefficient, $A$ is the projected area normal to the velocity, $\rho$ is the water density, and $v$ is the flow velocity. The peak $C_{T}$ was ascertained for each test.

For each test trial, the lowest speed that would cause at least one component of the cervical spine loading to reach an IARV was estimated. This was accomplished by solving Eq. (3) for the flow velocity $(v)$ using each IARV component (compression, tension, flexion, or extension) and assuming $C_{T}$ remained at its peak level throughout the water impact. For each test, the maximum of the relationship between drag force and cervical spine moment (essentially the peak effective moment arm) was used to convert IARV moments to force; this assumes that the helmet geometry was the controlling factor for the effective moment arm. For each impact configuration and helmet condition, the lowest speed across all tests was considered to be the speed to reach cervical spine IARV (that is, the lowest speed to produce a $3 \%$ chance of serious cervical spine injury).

\subsection{Statistical analysis}

A statistical analysis was conducted to compare the performance of the three different helmets versus each other and relative to the non-helmeted condition. Each metric was compared using $t$ tests with Bonferroni corrections for multiple comparisons to identify the specific pairs of test conditions for which the mean values were significantly different.

\section{Results}

A total of 55 tests were conducted with consistent ATD kinematics for each impact configuration; see Fig. 3 for frames from the high-speed video. The speed at water 
entry was $8.8 \pm 0.13 \mathrm{~m} / \mathrm{s}$ (range: $8.6-8.9 \mathrm{~m} / \mathrm{s}$ ). The full set of results are provided in Table 2. Across all test configurations, the head angular accelerations and likelihood of mTBI were less than $1000 \mathrm{rad} / \mathrm{s}^{2}$ and $1 \%$ and did not change significantly with water sport helmet use. Though water sports and bicycle helmets increased significantly $(p<0.05)$ many of the other injury metrics (such as HIC and cervical spine compression), the results for all metrics were below IARVs [18] and the angular accelerations associated with rotational brain injury $[19,20]$.

The total drag coefficients $\left(C_{T}\right)$ varied significantly depending on the impact configuration and the angle of water entry; see Table 2. Though the helmets increased the $C_{T}$ and reduced the speed necessary to cause injury for the Pelvis-First condition, only the bicycle helmet produced a significantly higher $C_{T}$ value and lower predicted cervical spine injury speed compared to the nonhelmeted condition. For all Pelvis-First water impacts, the lowest speed related to cervical spine injury was $35 \mathrm{~m} / \mathrm{s}$ (126 kph).

\section{Discussion}

Others have measured directly the head accelerations of water skiers during non-injurious falls [13]. In their tests, water skiers were towed at $9 \mathrm{~m} / \mathrm{s}$, instructed to release the ski rope, and fell forward. Though Lyons et al. [13] reported that their data were highly variable, the mean head accelerations reported were between 2.5 and 2.6 Gs and the high end of the $95 \%$ confidence interval was 5.2 Gs. Our head acceleration data were higher for our Head-First test scenarios; our tests with Helmet A (the same helmet model used in [13]) produced mean head accelerations of $7.1 \mathrm{Gs}$ and 4.9 Gs for the Head-First, Shallow and Inclined tests. The differences may come from a higher head to water impact speed in the present testing (it was not reported in [13]), differences in water entry kinematics, and differences between the ATD model and a water skier. These data suggest that our tests are likely representative of (or are more severe than) impacts for a water skier in a contrived forward fall while skiing at $9 \mathrm{~m} / \mathrm{s}$.

In this present study, the peak linear head acceleration and HIC remained less than $4 \%$ and $1 \%$ of Injury Assessment Reference Values (IARVs) [18] and the associated
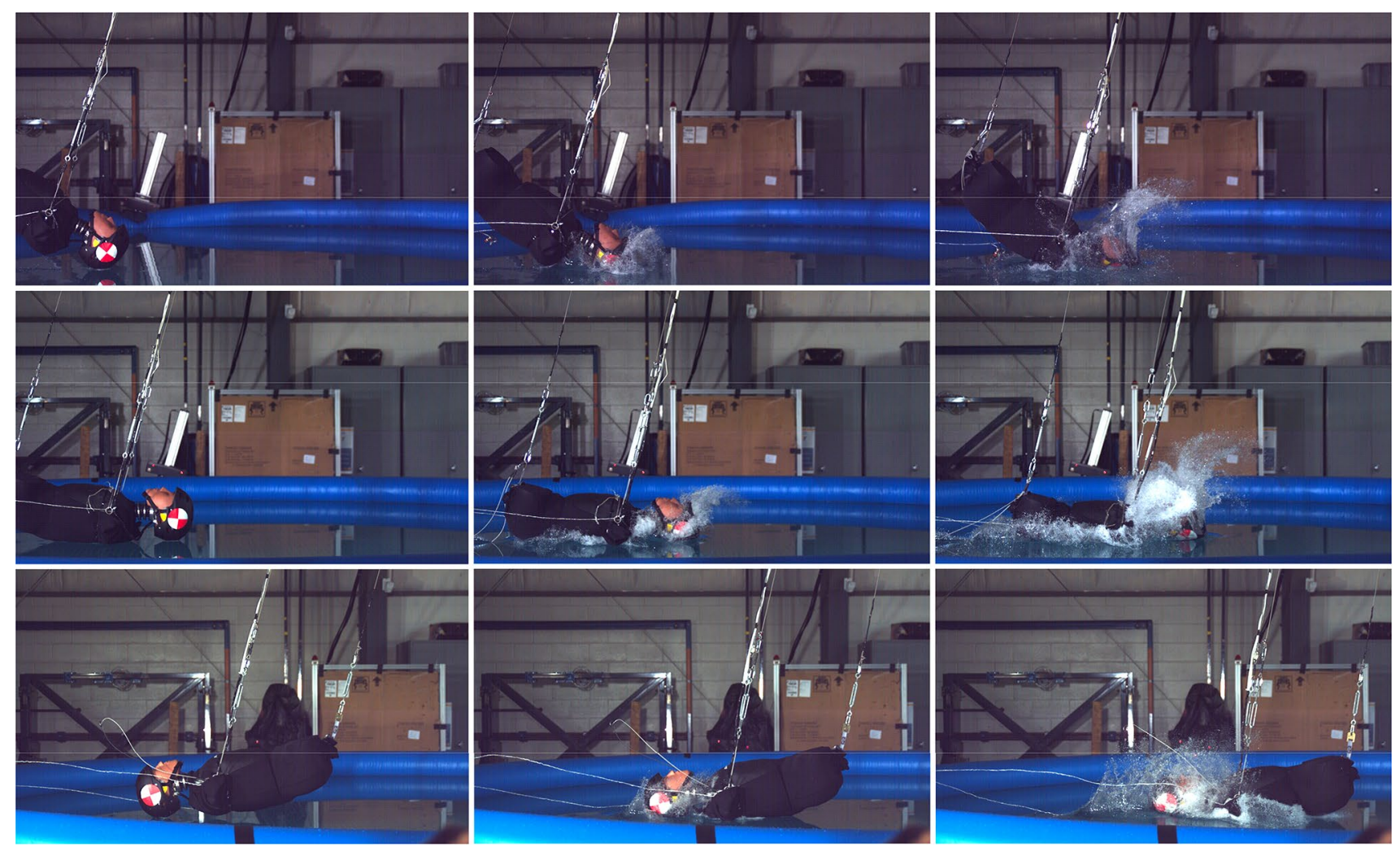

Fig. 3 Still frames from the high-speed video of tests with Helmet A: (top row) Head-First, inclined; (middle row) Head-First, shallow; and, (bottom row) the Pelvis-First conditions. For each row, the left frame is the initiation of water entry $(0 \mathrm{~ms})$, the center frame is at $25 \mathrm{~ms}$, and the right frame is at $50 \mathrm{~ms}$ 


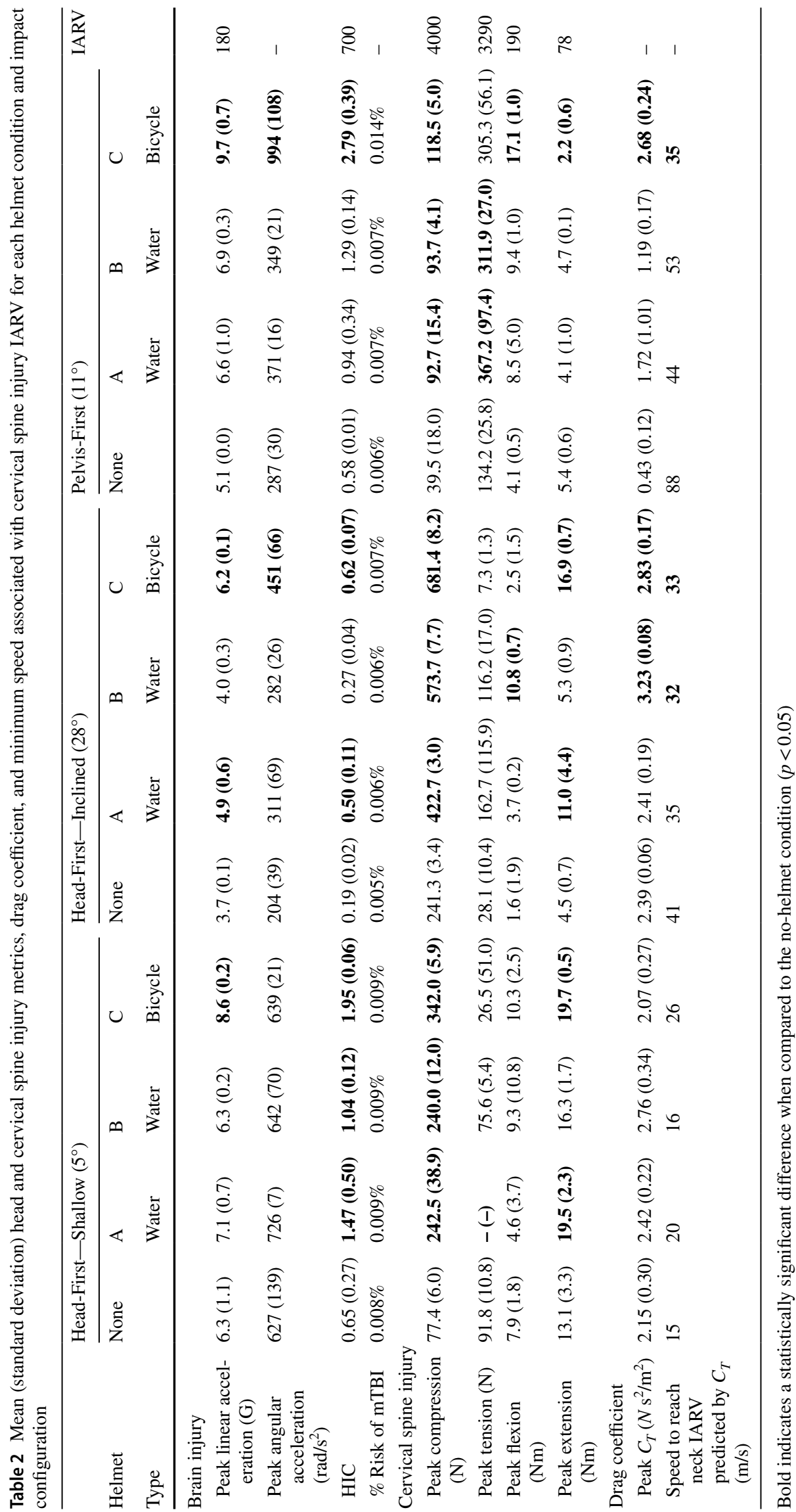


injury likelihoods were low (under 1\%) both with and without a water sports helmet. Similarly, the peak cervical spine compression, tension, flexion, and extension remained less than $14 \%, 11 \%, 6 \%$, and $25 \%$ of the cervical spine IARVs [18] and the associated likelihoods for serious injury were low (under 1\%) both with and without a water sports helmet. While some of the differences between tests with and without a water sports helmet were statistically significant $(p<0.05)$, the water sports helmets did not increase meaningfully the likelihood of head or cervical spine injury during water entry at the speed tested. For example, though Helmet A had a higher mean linear head acceleration than the non-helmeted tests in the Head-First, Inclined $\left(28^{\circ}\right)$ configuration $(p<0.05)$, the peak linear acceleration $(4.9 \mathrm{Gs})$ and HIC $(0.5)$ remained well below the IARVs $(180 \mathrm{Gs}$ for linear acceleration and 700 for HIC); all head injury metrics remained well below the $1 \%$ chance for severe [18] or mild traumatic [21] brain injury. Similarly, Helmet B had a higher mean peak cervical spine flexion moment $(10.8 \mathrm{Nm})$ than the non-helmeted tests $(1.6 \mathrm{Nm})$ in the Head-First, Inclined $\left(28^{\circ}\right)$ configuration $(p<0.05)$, but the flexion moments all remained well below the IARV (190 Nm); all cervical spine injury metrics remained well below the $1 \%$ chance of serious injury (using [18]). Similar results were found for the other comparisons between statistically significant injury metrics. These data suggest that head and neck injuries are unlikely during water skiing and wakeboarding falls into water, both with and without a water sports helmet; low injury risk is not $0 \%$ injury risk and it is still possible to sustain an injury. This is consistent with the epidemiologic data; while the epidemiologic data show that prevalence for head and neck injuries in wakeboarding and water skiing is high $(47.9 \%$ and $24.6 \%$ from [5]), the incidences of head and neck injuries in wakeboarding and water skiing were low ( 0.39 and 0.55 per 100,000 individuals from [5]). Similar epidemiologic results have been reported for concussions in wakeboarding and water skiing [5]. The data presented above do not support the supposition that helmets increase the likelihood of injury by an appreciable amount at the speed tested (that is typical for recreational water sports).

For the helmeted tests, the lowest water impact speed predicted to produce loads above the cervical spine IARV was $16 \mathrm{~m} / \mathrm{s}$ (58 kph), for Helmet B in the Head-First, Shallow configuration (see Table 2). Interestingly, the Head-First, Shallow tests with no helmet also produced large extension moments with low compressive loads that generated a lower predicted speed to exceed the extension moment IARV $(15 \mathrm{~m} / \mathrm{s}$ or $64 \mathrm{kph})$ compared to the helmeted conditions. There is no obvious reason (such as geometric differences, helmet-to-head motions, surface roughness, etc.) why Helmet B and the no-helmet conditions would have larger effective moment arms than Helmets A and C. It was expected that the larger size, visor, and complex posterior geometry of Helmet $\mathrm{C}$ (the bicycle helmet) would increase the effective (total) drag coefficient $\left(C_{T}\right)$ more than the smaller, rounded water sports helmets. Surprisingly, Helmet $\mathrm{C}$ produced lower $C_{T}$ values and higher speeds to reach IARVs when compared to one or both water sports helmets for the Head-First configurations. This was not always the result, as Helmet $\mathrm{C}$ produced a significantly larger $C_{T}$ and lower speeds for predicted cervical spine injury compared to the water sports helmets in the Pelvis-First tests. Despite the differences in helmets, there was no statistical difference for the lowest water impact speed predicted to produce loads above the cervical spine IARV between the helmeted and non-helmeted conditions for the Head-First, Shallow configuration.

The water impact speed used in the present study was similar to the pre-fall water skier speed used in previous testing [13] and the pre-fall speeds at cable-tow wakeboard parks $(9 \mathrm{~m} / \mathrm{s})$. Higher water impact speeds are certainly possible with higher towed speeds. Previous research reported that water skiers can reach on average $127 \%$ of the boat speed [22]. For a typical water ski competition boat speed of $16 \mathrm{~m} / \mathrm{s}(58 \mathrm{kph})$, a water skier would likely reach $20 \mathrm{~m} / \mathrm{s}$ (72 kph); this is consistent with the speeds reported in other research [23]. For this testing, water entry speed was limited by the height of the test facility. The lowest speeds predicted to exceed the cervical spine IARVs in our tests (Helmet B and no helmet for the Head-First, Shallow configuration) are within the range of expected speeds $(8-21 \mathrm{~m} / \mathrm{s})$ for competition water sports participants; this is consistent with the injuries reported in the epidemiologic studies $[5,6]$. The data from the present testing do not suggest that wearing a water sports helmet would increase the injury likelihood by more than $1 \%$.

One idea to reduce the bucketing effect is to increase the number and size of the helmet vents. This could facilitate outflow from the space between the head and helmet. The helmets used in the testing spanned the range of venting available in water sports helmets: Helmet A had the most venting (by area and number) available commercially and Helmet $\mathrm{B}$ had the least venting (no vents at all). Counterintuitively, the $C_{T}$ values for Helmet A $(1.72 \pm 1.01)$ were larger than those for Helmet B $(1.19 \pm 0.17)$ in our Pelvis-First testing (the tests trying to maximize bucketing), but this difference was not significant $(p=1.0)$. Helmet $\mathrm{C}$ (the bicycle helmet) produced significantly larger $C_{T}$ values $(2.68 \pm 0.24)$ and lower injury speeds when compared to the other helmets in the Pelvis-First configuration. These differences may have been generated by the shape at the occipital edge of the helmet and the posterior protrusions, and not the venting. While a water sport helmet affected the loads produced in the Pelvis-First water impacts, the predicted speed needed to reach injury level $(44 \mathrm{~m} / \mathrm{s})$ was well above the highest speeds in water skiing and wakeboarding (under $22 \mathrm{~m} / \mathrm{s}$ ) [22, 23]. 
Though water sports helmets offer protection in head contacts with hard objects [24, 25], there may be room for improvement. The only water sports helmet standard (EN 1385 [26]) requires the headform acceleration remain under 250 Gs when the EN 960 size $\mathrm{J}$ headform is dropped onto a flat, steel anvil at $2.53 \mathrm{~m} / \mathrm{s}(9.1 \mathrm{kph})$. Compared to other recreational sport helmets, water sport helmets produce higher accelerations for the same drop parameters [27]. Future development work on new liner materials and helmet geometry should consider carefully how these changes may influence performance during water only contacts.

\section{Limitations}

As with all testing, our water sports fall model had limitations, including the ATD used. The geometry and stiffness of the Hybrid-III neck are different from a live person and unbiased neckforms [28]. Similarly, 50th percentile HybridIII headforms do not represent realistically the geometry and helmet fit for people [29, 30]. The larger diameters and irregularities of a live person's head would be expected to increase the effective (total) drag coefficient $\left(C_{T}\right)$ in unhelmeted falls and would reduce the differences between unhelmeted and helmeted tests. While other headforms may be more biofidelic than the Hybrid-III, such as novel human head surrogates [31] or the NOCSAE headforms, their availability or the ability to waterproof the sensors made them too difficult to use in this testing.

Though the water entry speed tested was similar to the speeds of wakeboarders in a cable-tow park and recreational water skiers towed behind a boat, competition-level water skiers can travel faster and impact the water at higher speeds. The helmet effects at higher speeds were considered by ascertaining the $C_{T}$ (from Eq. (3)) for each test and using it to calculate the kinematics and kinetics of higher water impact speeds. These calculations assumed that the $C_{T}$ would not change with water impact speed, water temperature, salinity, etc. $C_{T}$ is dependent on water density $(\rho)$, the geometric drag coefficient $\left(C_{D}\right)$, and projected area normal to the velocity $(A)$. Because water is generally incompressible, it is not expected that $\rho$ would change much for bodies of water in which people would water ski and wakeboard and, therefore, not alter $C_{T}$ by much. Both $A$ and $C_{D}$ are functions of the helmet geometry and $C_{D}$ is also dependent on Reynolds number (that is dependent on $v$ ). Because the impact speed could not be increased (limited by test facility height), an auxiliary set of 24 tests were conducted at lower water entry speeds of 4.5 and $6.7 \mathrm{~m} / \mathrm{s}$ (at lower drop heights) to examine the effects of impact speed on the peak $C_{T}$ value. The peak $C_{T}$ value for these lower speed tests were within $7 \%$ of the values determined for the higher-speed tests (and within one standard deviation) and not consistently higher or lower than the means determined at higher speed. Because the $C_{T}$ values did not change much over the range of speeds tested, using these $C_{T}$ values to estimate helmet effects at higher speeds may be reasonable.

Our model did not capture all elements of a water sports fall. In falls where an edge catch occurs, a water skier or wakeboarder can rotate rapidly from a nearly upright position in a short fraction of a second to enter the water at the angles tested; in these cases, the water entry speed could be higher than those tested. It was not possible to model this pre-impact angular velocity with our pendulum system.

\section{Conclusions}

Though water sports helmets did increase some injury metrics (such as head acceleration, HIC, and cervical spine compression), the metrics remained below IARVs and the likelihoods of injury remained below $1 \%$. Head-first water impacts were more likely to produce cervical spine injuries when compared to falls that produce bucketing. If additional development on water sports helmet standards or design is undertaken, the data above do not support sacrificing impact attenuation for additional venting to prevent bucketing. The testing does not support the supposition that water sports helmets would increase the likelihood of head or neck injury in a typical fall during recreational water sports.

\section{Compliance with ethical standards}

Conflict of interest This research did not receive any specific grant from funding agencies in the public, commercial, or not-for-profit sectors. The authors have conducted unrelated research for water sports industry associations.

Open Access This article is licensed under a Creative Commons Attribution 4.0 International License, which permits use, sharing, adaptation, distribution and reproduction in any medium or format, as long as you give appropriate credit to the original author(s) and the source, provide a link to the Creative Commons licence, and indicate if changes were made. The images or other third party material in this article are included in the article's Creative Commons licence, unless indicated otherwise in a credit line to the material. If material is not included in the article's Creative Commons licence and your intended use is not permitted by statutory regulation or exceeds the permitted use, you will need to obtain permission directly from the copyright holder. To view a copy of this licence, visit http://creativecommons.org/licenses/by/4.0/.

\section{References}

1. Sports and Fitness Industries Association (2017) Sports, Fitness, and leisure activities topline participation report. Sports and Fitness Industries Association, Silver Spring 
2. Carson WG (2004) Wakeboarding injuries. Am J Sports Med $32: 164-173$

3. Loughlin S (2013) Investigation of injuries occurring within competitive water skiing in the UK. Int J Exerc Sci 6:29-42

4. Hostetler SG, Hostetler TL, Smith GA, Xiang H (2005) Characteristics of water skiing-related and wakeboarding-related injuries treated in emergency departments in the United States, 2001-2003. Am J Sports Med 33:1065-1070

5. Baker J, Griffin R, Brauneis P, Rue L III, McGwin G Jr (2010) A comparison of wakeboard-, water skiing-, and tubing-related injuries in the United States, 2000-2007. J Sports Sci Med 9:92-97

6. Schofer MD, Hrabal SA, Timmesfeld N, Fuchs-Winkelmann S, Patzer T (2010) Cable wakeboarding, a new trendy sport: analysis of injuries with regard to injury prevention. Scand J Med Sci Sports 22:583-583

7. Hummel G, Gainor BJ (1982) Water skiing-related injuries. Am J Sports Med 10:215-218

8. Chia JK, Goh KY, Chan C (2000) An unusual case of traumatic intracranial hemorrhage caused by wakeboarding. Pediatr Neurosurg 32:291-294

9. Kluger N, Jegou M-H (2016) Severe neck lacerations during cable skiing in a child. La Presse Med 45:540-541

10. Yliniemi EM et al (2009) Dynamic tensile failure mechanics of the musculoskeletal neck using a cadaver model. J Biomech Eng 131:051001

11. Taylor RK, Robbins R, Fuller PM, Savander BR (2000) Impact loads experienced by the cervical spine during high-speed water entry. In: Proc. IRCOBI, International Research Council on Biomechanics of Injury, 28, 479-481

12. Scheer D, Karami G, Ziejewski M (2015) Effects of helmet surface geometry on head acceleration in high velocity water sports. Procedia Eng 112:485-490

13. Lyons JC, Pollard M, Goodman G, Cooney TE (2012) The severity of impacts during low-speed water skier falls. Open Sports Med J 6:29-33

14. Bailly N, Llari M, Donnadieu T, Masson C, Arnoux P-J (2017) Head impact in a snowboarding accident. Scand J Med Sci Sports 27:964-974. https://doi.org/10.1111/sms.12699

15. SAE J211 (2014) Instrumentation for impact test-part 1-electronic instrumentation. SAE Standard. SAE International. https:// www.sae.org/standards/content/j211/1_201403/

16. Bussone WR, Bove RT, Thomas R, Richards D, Prange MT (2010) Six-Degree-of-Freedom accelerations: linear arrays compared with angular rate sensors. SAE Technical Paper 2010-011017. https://doi.org/10.4271/2010-01-1017

17. Eppinger R, Sun E, Bandak F, Haffner M, Khaewpong N, Maltese M, Kuppa S, Nguyen T, Takhounts E, Tannous R, Zhang A, Saul R (1999) Development of improved injury criteria for the assessment of advanced automotive restraint systems-II. NHTSA, Washington, DC. https://one.nhtsa.gov/cars/rules/rulin gs/80g/80gii.html

18. Mertz H, Irwin A, Prasad P (2003) Biomechanical and scaling bases for frontal and side impact injury assessment reference values. Stapp Car Crash J 47:155-188

19. Depreitere B et al (2005) Cerebral bridging vein rupture in humans. In: Gilchrist MD (ed) IUTAM symposium on impact biomechanics: from fundamental insights to applications. Solid mechanics and its applications, vol 124. Springer, Dordrecht
20. Depreitere B et al (2006) Mechanics of acute subdural hematomas resulting from bridging vein rupture. J Neurosurg 104:950-956. https://doi.org/10.3171/jns.2006.104.6.950

21. Rowson S, Duma SM (2013) Brain injury prediction: assessing the combined probability of concussion using linear and rotational head acceleration. Ann Biomed Eng 41:873-882

22. Runciman RJ (2001) Water-skiing biomechanics: a study of intermediate skiers. Proc Inst Mech Eng Part P J Sports Eng Tech 225(4): 231-239. https://doi.org/10.1177/1754337111403693

23. Bray-Miners J, Runciman RJ, Monteith G, Groendyk N (2014) Biomechanics of slalom water skiing. Proc Inst Mech Eng Part P J Sports Eng Tech 229:47-57

24. Stepan L, Scher I, Thomas R (2010) Protective capabilities of a water sports helmet for boom-to-head impacts during sailing. In: Proceedings of the ASME 2010 summer bioengineering conference. ASME 2010 summer bioengineering conference, Parts A and B, Naples, Florida, USA, 16-19 June 2010. ASME, pp. 305-306. https://doi.org/10.1115/SBC2010-19717

25. Duma B, Begonia M, Tyson A, Charron C, Duma S (2019) Water sport head injuries: ability of helmets to reduce head impact accelerations. In: Proceedings of the ASME 2019 summer biomechanics, bioengineering, and biotransport conference, Seven Springs, Pennsylvania, USA, 25-28 June 2019. \#P031. https://archive.sb3c. org

26. European Standard BS EN 1385:1998/A1:2005 (2005) Helmets for canoeing and white water sports

27. Thom D (2014) ASTM F08.53 low velocity impact data. Presented and submitted to ASTM F08.53.

28. Walsh ES, Kendall M, Post A, Meehan A, Hoshizaki TB (2018) Comparative analysis of hybrid III neckform and an unbiased neckform. Sports Eng 21(4):479-485

29. Liu K, Aponte DI, Greencorn DJ, Robbins SM, Pearsall DJ (2019) Are headforms a poor surrogate for helmet fit?. Proc Inst Mech Eng Part P J Sports Eng Tech. https://doi.org/10.1177/17543 37119882836

30. Liu K, Greencorn DJ, Aponte DI, Robbins SM, Pearsall DJ (2019) Comparison of surrogate 50th percentile human headforms to an adult male sample using three-dimensional modeling and principal component analysis. Proc Inst Mech Eng Part P: J Sports Eng Technol 233(3):432-442. https://doi.org/10.1177/1754337119 840848

31. Petrone N, Candiotto G, Marzella E, Uriati F, Carraro G, Bäckström M, Koptyug A (2019) Feasibility of using a novel Instrumented Human head surrogate to measure helmet, head and brain kinematics and intracranial pressure during multidirectional impact tests. J Sci Med Sport 22(S1):S78-S84. https://doi. org/10.1016/j.jsams.2019.05.015

32. European Standard BS EN 1078:2012+A1:2012 (2013) Helmets for pedal cyclists and for users of skateboards and roller skates

Publisher's Note Springer Nature remains neutral with regard to jurisdictional claims in published maps and institutional affiliations. 